\title{
Combining experiences of race gaming and natural driving affects gaze location strategy in simulated context
}

Gisele C. Gotardi ${ }^{\mathrm{a}, \mathrm{c}}$, Gabriel K. Kuga ${ }^{\mathrm{a}}$, Rafael O. Simão ${ }^{\mathrm{a}, \mathrm{c}}$, Matheus B. Brito ${ }^{\mathrm{a}, \mathrm{c}}$,

Gabriel P. Paschoalino ${ }^{\mathrm{a}, \mathrm{c}}$, Gustavo A. Silva ${ }^{\mathrm{b}, \mathrm{c}}$, Fabio A. Barbieri, ${ }^{\mathrm{a}, \mathrm{d}}$, Paula F.

Polastri $^{\mathrm{a}, \mathrm{c}}$, Paulo Schor ${ }^{\mathrm{e}}$, Martina Navarro ${ }^{\mathrm{f}}$, Sergio T. Rodrigues ${ }^{\mathrm{a}, \mathrm{b}, \mathrm{c}}$

${ }^{a}$ Graduate program in Human Movement Science, Department of Physical Education,

Faculty of Sciences, São Paulo State University, Bauru, Brazil; ${ }^{b}$ Graduate program in

Design (Ergonomics), Faculty of Architecture, Arts, and Communication, São Paulo State

University, Bauru, Brazil; ' Laboratory of Information, Vision, and Action (LIVIA),

Department of Physical Education, Faculty of Sciences, São Paulo State University, Bauru,

Brazil; ' Human Movement Research Laboratory (MOVI), Department of Physical Education,

Faculty of Sciences, São Paulo State University, Bauru, Brazil; ${ }^{e}$ Department of

Ophthalmology and Visual Sciences, Paulista School of Medicine, Federal University of São

Paulo, São Paulo, Brazil; ${ }^{f}$ Department of Sport and Exercise Science, Faculty of Science,

University of Portsmouth, Portsmouth, United Kingdom.

Corresponding author: Sergio T. Rodrigues, São Paulo State University, Av. Eng. Luiz

Edmundo Carrijo Coube, 14-01, Vargem Limpa, Bauru, SP, Brazil, postal code 17033-360, phone +55 14 31039617, email: srodrigu@ fc.unesp.br

Gisele Chiozi Gotardi (gcgotardi@gmail.com); Gabriel K. Kuga (xgabriel@gmail.com); Rafael O.

Simão (rafaelosimao@gmail.com); Matheus B. Brito (matheus.belizario@ hotmail.com); Gabriel P.

Paschoalino (gpaschoalino@ hotmail.com); Gustavo A. Silva (obscuremetaller@gmail.com); Fabio A,

Barbieri (barbieri@fc.unesp.br); Paula F. Polastri (paulafp@fc.unesp.br); Paulo Schor

(pschor@pobox.com); Martina Navarro (martina.navarro@port.ac.uk).. 


\section{Combining experiences of race gaming and natural driving affects gaze location strategy in simulated context}

The aims of the study were to investigate the effects of race gaming experience in playing racing video games on gaze behaviour and performance of drivers and the effects of natural driving experience on gaze behaviour and performance of gamers. Thirty participants, divided into drivers-gamers, drivers-non-gamers and non-driversgamers, were asked to drive in a race circuit as fast as possible while their eye movements were recorded. Drivers-gamers spent more time looking to the lane than non-drivers-gamers. Furthermore, drivers-gamers performed greater number of fixations towards the speedometer and showed faster performance in the racing task than the drivers-non-gamers. Combining natural driving and race gaming experiences changes the gaze location strategy of drivers.

Keywords: racing video games; ergonomics; visual search; virtual environment

Practitioner Summary: Racing video games practitioners have high propensity to exhibit attitudes and intentions of risky driving behaviour. Combining natural driving and race gaming experiences affects gaze behaviour strategy of drivers.

\section{Introduction}

Playing action video games modifies visual attention and oculomotor performance. Video game players have shown faster reaction time to detect peripheral targets and faster stimulus-response mapping in both easy and difficult visual search tasks (Castel, Pratt, and Drummond 2005; Dye, Green, and Bavelier 2009), better performance in ignoring distractors and an improved attentional switching ability (Green and Bavelier 2003; Green and Bavelier 2007), as well as an enhanced visual short-term memory (McDermott, Bavelier, and Green 2014) than non-video game players. In addition, experienced gamers also have demonstrated 
shorter saccadic reaction time and higher saccadic peak velocities than non-gamers (Mack and Ilg 2014; West, Al-Aidroos, and Pratt 2013).

In the last decade, growth in video game usage has been observed worldwide. In Brazil, 56 million people regularly use video games as their favourite pastime; racing games appear as a very popular game type (IBOPE 2014). This broad interest can be due to the fact that these games offer realistic interactions for their players, simulating highly complex traffic scenarios, what would represent the visual experience of drivers in the natural, out-oflaboratory world (Beullens, Roe, and Van Den Bulck 2011; Ciceri and Ruscio 2014). Predrivers, such as pedestrians and cyclists, are particularly active road users that intend to become drivers (Gatersleben and Haddad 2010) and possibly interact with complex traffic environments through driving simulators and racing video games. However, there is a relationship between video game playing and adolescents' attitudes and intentions to exhibit risky driving behaviour in the future (Beullens, Roe, and Van Den Bulck 2011). Thus, the impact of playing video games on perceptual and motor performance of both drivers and predrivers (i.e., typically racing video gamers) has been a research topic in focus in human factors scientific field.

Recent studies with virtual environment (e.g., action video games, simulators, and virtual reality) have emerged as an alternative approach to study and understand skilled performance in sports (Craig 2014; Neumann et al. 2017), physical rehabilitation programs for vulnerable populations such as patients with Parkinson's disease and cerebral palsy (Howard 2017), and motor learning process (Pasco 2013). Particularly, drivers in their earliest years of driving license are more susceptible to be involved in traffic accidents (Clarke, Ward, and Truman 2005); gaze behaviour analyses have showed crucial differences between experienced and novice drivers (Underwood, Crundall, and Chapman 2007; Underwood et al. 2003). According to the World Health Organization, about 1.25 million 
people die by traffic accident and between 20 to 50 million victims become disabled by nonfatal crashes each year worldwide (World Health Organization 2016). The Institute of Applied Economic Research of Brazil (IPEA) recently revealed that all traffic accidents in 2014 had a total cost for Brazilian Government estimated in $\mathrm{R} \$ 40$ billion (Instituto de Pesquisa Econômica Aplicada 2015). These economic losses are consequences of high costs in rehabilitation treatments, reduction of the labour productivity, investigation of accident causes and others and represent a serious issue of Public Health and Economic Management that needs to be attenuated.

Driving experience plays a critical role in driving. It has been shown that experienced drivers perform a greater number of shorter fixations with a wider horizontal spread across the visual scene when compared to the novice ones (Crundall and Underwood 1998; Konstantopoulos, Chapman, and Crundall 2010), indicating a faster processing time and, consequently, a superior steering control performance. For this reason, the understanding of driver's learning process is an important topic in cognitive ergonomics research and a relevant issue to prevent road accidents. Ciceri and Ruscio (2014) recorded eye movements of experienced- and non-drivers (participants who were regularly players of racing video games) in both natural and simulated scenarios with the same type of road interactions. Drivers-gamers performed fixations more often, with longer durations, towards the relevant portions of the visual scene (i.e., lane, rearview mirrors, and speedometer), and moved their eyes horizontally in a wider spread than non-drivers-gamers. In addition, non-drivers-gamers ignored traffic road signs and potential areas of interactions (e.g. crosswalk and roundabouts) (Ciceri and Ruscio 2014). The authors suggested that visual search strategy of non-driversgamers was poorer and unsafe when compared to drivers-gamers. On the other hand, it is unclear whether the specific gaze strategy demonstrated by the drivers-gamers, in comparison 
with non-drivers-gamers, was influenced by natural driving experience or video game practice.

The aim of this study was to investigate the effects of experience in playing racing video games on gaze behaviour and performance of drivers during a simulated racing task. An additional goal was to determine the effects of experience in driving under natural circumstances on racing gamers' visuomotor performance. It was hypothesized that the most experienced participants, the drivers-gamers, would exhibit a gaze strategy that combines the smallest relative number of fixations, the largest relative fixation durations, mainly directed to the race lane area of interest, and the largest variance of fixations, particularly in the vertical axis, with the smallest total race time as compared to driver-non-gamer and nondrivers-gamers. Differences between drivers-gamers and drivers-non-gamers were expected to represent effects of the experience in playing racing video games while differences between drivers-gamers and non-drivers-gamers were expected to represent effects of the experience due to licensing process and natural driving practice.

\section{Method}

\section{Participants}

Thirty young adults voluntarily participated in this experiment and were divided in three experimental groups according to their driving experience in natural and virtual environments. Ten drivers-gamers $(\mathrm{DG}, 26.6 \pm 2.3$ years old, $84.6 \pm 18.9 \mathrm{~kg}, 176.9 \pm 3.7$ $\mathrm{cm}$ ), with natural, real-world driving experience of at least four years and who regularly play racing video games at the minimum frequency of three times per week with one hour per session; ten drivers-non-gamers (DNG, $29.2 \pm 5.0$ years old, $68.4 \pm 22.4 \mathrm{~kg}, 166.4 \pm 11.6$ $\mathrm{cm}$ ), with natural, real-world driving experience of at least four years and without video game experience in any type of game; and ten non-drivers-gamers (NDG, $20.9 \pm 3.8$ years old, 65.9 
$\pm 10.7 \mathrm{~kg}, 168.4 \pm 6.9 \mathrm{~cm}$ ), who intend to become a driver (e.g., pre-drivers, Gatersleben and Haddad 2010) and regularly play racing video games at the minimum frequency of three times per week with one hour per session. Participants with normal or corrected vision, no sensorimotor, and neurological impairment were selected. Ethical approval for the study was obtained from the Local Committee and participants signed informed consent before starting the study.

\section{Apparatus}

Eye movements were recorded using Head-mounted Eye Tracker (model H6, Applied Science Laboratory, USA) at a sampling rate of $60 \mathrm{~Hz}$. This video-based analysis system of eye movements contains two micro-cameras, one film the left eye and other record the scene, attached to a headgear that was anatomically adjusted to the participant's head. For the eye video, pupil and corneal reflection centroids were identified and the vector between both of them were used to determine horizontal and vertical coordinates of eye position on scene video. The racing video game Gran Turismo (version 4) was run on PlayStation 2 (SONY) console and it was projected from multimedia BenQ (MX720) to a screen Projetelas (model Infinity) $204 \mathrm{~cm} \mathrm{x} 154 \mathrm{~cm}$. In order to provide a more realistic driving context to the experimental task, steering wheel and accelerator and brake pedals were used (Logitech model G-27). The steering wheel was attached to a regular table $($ width $=1.3 \mathrm{~cm} \times$ depth $=$ $59.5 \mathrm{~cm} \times$ height $=75 \mathrm{~cm}$ ) and pedals were set on the floor in front of the participant; distances between the participants and the accessories were individually defined to allow a comfortable driving posture. The racing video game Gran Turismo was configured in Arcade Mode, Time Trial, Original Circuits, Autumn Ring Mini, and Ford Ka’01. 


\section{Procedure}

Prior to start testing, participants were asked to take a seat and had eye tracker's headgear adjusted on their head. A nine-point calibration plan was projected on the TV screen and participants were asked to maintain their head as still as possible and to move their gaze from one point to another in ascending order. After calibration and prior to data collection, participants had three minutes to familiarize themselves with the racing game software and the experimental apparatus while driving. Furthermore, participants were asked to drive as fast as possible at the race circuit for three laps, with the absence of other vehicles. This racing task was performed twice for each participant while data were collected.

\section{Data analysis}

Gaze recordings were transferred to a PC (ASUS) running ASL Results Plus software (version 1.8.2.18, Applied Science Laboratory, USA) for further analysis with Areas of Interest (AOIs). AOIs were two-dimensional (2-D) regions defined in the viewing plane (e.g., scene video from Eye Tracker) in which the gaze fixation patterns (number and duration of fixations) were identified in relevant parts of the visual scene. The fixation criteria was as follow: fixation onset occurred when two times point of gaze standard deviation (95\% confidence interval) was less than one degree of visual angle (horizontal and vertical) over $100 \mathrm{~ms}$ (seven data samples); fixation offset occurred when three data samples deviated from initial fixation value by more than one degree of visual angle (horizontal and vertical). Four AOIs were defined (Figure 1): i) race lane, that provided the essential visual information to steering control (Land and Horwood 1995; Land and Lee 1994); ii) chronometer, that provided visual feedback about the duration (s) of each lap and the total duration (s) of each participant performance as a sum of the three laps; iii) speedometer, that provided visual feedback of the car speed throughout the trial; $i v)$ outside, all parts of the visual scene that 
were not included in other AOIs. Considering that each participant completed the driving task in different durations, relative fixations were calculated according to the specific trial duration of each participant; relative number of fixations (r-NUMFIX) in each AOI (unit/min) was calculated by dividing the total number of fixations in each AOI by the total trial time (converted from seconds to minutes). In addition, the relative fixations duration ( $\mathrm{r}-$ DURFIX) in each AOI (\%) were measured by dividing the total fixation duration in each AOI by the total trial time multiplied by one hundred, expressing the percentage of the total trial time that participants remained with the eyes stationary on each AOI.

Horizontal and vertical variances of fixation locations (pixels) were calculated to express the range of visual scanning strategy employed by drivers (Crundall, Underwood, and Chapman 1999; Crundall and Underwood 2011). Horizontal and vertical axes of fixation positions were exported as text files in a matrix of two columns by the number of rows equivalent to the number of fixations found in each trial. The square root of standard deviations of the fixation positions in both axes were calculated using Matlab (Mathworks, 7.10.0.499). The scene video of the Eye Tracker provided the recording of performance. The total race time (s) was measured for each participant in each trial by summing the race time in each of the three laps.

[Figure 1 near here]

\section{Statistical analysis}

In order to specify the contribution of each experience factor of interest such as natural driving and race gaming, the statistical analysis was divided in two tests. Data were tested for effect of driving experience on gaze behaviour in a racing video game environment; dependent variables were submitted to a one-way Analysis of Variance (ANOVA) with group main effect (DG, NDG). Additionally, gaming experience on gaze 
behaviour in a racing video game environment was investigated; a one-way ANOVA with group main effect (DG, DNG) was conducted. The analyses for r-NUMFIX and r-DURFIX were run for each AOI. Tukey Honestly Significant Difference tests, Greenhouse-Geisser degrees of freedom adjustments, and Bonferroni multiple-comparison probability adjustments were conducted when necessary. The value alpha was 0.05. Effect sizes were calculated using Partial Eta Squared with 0.02 or less, approximately 0.13 , and 0.26 or more, representing small, medium, and large effect sizes, respectively (Cohen 1988).

\section{Results}

A complete overview of means and standard deviations of the all dependent variables for both driving and gaming experience analyses are provided in Table 1.

[Table 1 near here]

\section{Driving experience: drivers-gamers vs. non-drivers-gamers}

For the r-NUMFIX, the analysis revealed no effect of group on all AOIs: outside, $\mathrm{F}(1,18)=1.757, \mathrm{p}=.202, \eta_{\mathrm{p}}{ }^{2}=.089 ;$ race lane, $\mathrm{F}(1.18)=.430, \mathrm{p}=.520, \eta_{\mathrm{p}}{ }^{2}=.023 ;$ chronometer, $\mathrm{F}(1,18)=1.844, \mathrm{p}=.191, \eta_{\mathrm{p}}{ }^{2}=.093$; and speedometer, $\mathrm{F}(1,18)=.168, \mathrm{p}=$ $.687, \eta_{\mathrm{p}}{ }^{2}=.009$. For the r-DURFIX, the ANOVA one-way revealed a main effect of group on the AOI race lane, $\mathrm{F}(1,18)=5.336, \mathrm{p}=.033, \eta_{\mathrm{p}}{ }^{2}=.229$. The DG spent more time performing fixations towards the race lane than NDG (Figure 2). There was no group main effect for the r-DURFIX on other AOIs: outside, $\mathrm{F}(1,18)=.739, \mathrm{p}=.401, \eta_{\mathrm{p}}{ }^{2}=.039$; chronometer, $\mathrm{F}(1,18)$ $=2.215, \mathrm{p}=.154, \eta_{\mathrm{p}}{ }^{2}=.110 ;$ and speedometer, $\mathrm{F}(1,18)=.093, \mathrm{p}=.764, \eta_{\mathrm{p}}{ }^{2}=.005$. For the horizontal and vertical variance of fixations, the analyses revealed no main effect of group, $\mathrm{F}(1,18)=.040, \mathrm{p}=.843, \eta_{\mathrm{p}}{ }^{2}=.002$ and $\mathrm{F}(1,18)=1.676, \mathrm{p}=.212, \eta_{\mathrm{p}}{ }^{2}=.085$, respectively. 


\section{Gaming experience: drivers-gamers vs. drivers-non-gamers}

For the r-NUMFIX, the analysis revealed a main effect of group on the AOI speedometer, $\mathrm{F}(1,18)=6.424, \mathrm{p}=.021, \eta_{\mathrm{p}}{ }^{2}=.263$. The DG showed a greater numbers of fixations towards speedometer than DNG during the racing task. There was no group main effect for the r-NUMFIX on other AOIs: outside, $\mathrm{F}(1,18)=.135, \mathrm{p}=.717, \eta_{\mathrm{p}}{ }^{2}=.007$; race lane, $\mathrm{F}(1,18)=.004, \mathrm{p}=.952, \eta_{\mathrm{p}}{ }^{2}=.000$; and chronometer, $\mathrm{F}(1,18)=.621, \mathrm{p}=.441, \eta_{\mathrm{p}}{ }^{2}=$ .033. For the r-DURFIX, the analyses revealed no effect of group on all AOIs: outside, $\mathrm{F}(1,18)=.296, \mathrm{p}=.593, \eta_{\mathrm{p}}^{2}=.016 ;$ race lane, $\mathrm{F}(1.18)=.739, \mathrm{p}=.401, \eta_{\mathrm{p}}{ }^{2}=.039$ chronometer, $\mathrm{F}(1,18)=.137, \mathrm{p}=.716, \eta_{\mathrm{p}}{ }^{2}=.008$; and speedometer, $\mathrm{F}(1,18)=4.230, \mathrm{p}=$ $.055, \eta_{\mathrm{p}}{ }^{2}=.190$. For the horizontal and vertical variance of fixations, the analyses revealed no effect of group, $\mathrm{F}(1,18)=.011, \mathrm{p}=.918, \eta_{\mathrm{p}}{ }^{2}=.001$ and $\mathrm{F}(1,18)=2.543, \mathrm{p}=.128, \eta_{\mathrm{p}}{ }^{2}=.124$, respectively. Regarding to the performance, the analysis revealed a group main effect for the total race time, $\mathrm{F}(1,18)=6.892, \mathrm{p}=.017, \eta_{\mathrm{p}}{ }^{2}=.277$. The DG performed the racing task faster than the DNG, showing a reduced total race time. The statistically significant differences found for both r-NUMFIX on the speedometer and the total race time between the groups (Figure 3) indicate that gaming experience increased the number of fixations towards speedometer and reduced the total race time of drivers in a racing video game environment.

[Figure 3 near here]

\section{Discussion}

The aim of this study was to investigate the effects of combining experiences of natural driving and race gaming on gaze behaviour and performance of drivers during a simulated racing task. Differences between drivers-gamers and drivers-non-gamers were expected to represent effects of the experience in playing racing video games while 
differences between drivers-gamers and non-drivers-gamers were expected to represent effects of the experience due to natural driving practice. It was hypothesized that the most experienced participants, the drivers-gamers, would exhibit a task-specific gaze location strategy that combines the smallest relative number of fixations and the largest relative fixation durations, mainly directed to relevant areas of the visual scene, with the shorter total race time (i.e., faster performance). The results revealed that the drivers-gamers spent more time performing fixations towards the race lane than non-drivers-gamers; the drivers-gamers also showed a greater relative number of fixations in the speedometer and performed faster the racing task as compared to the drivers-non-gamers. In the current study, the approach was focusing on the combined driving-gaming experience; the contribution of each factor, driving and gaming experiences, was separately considered.

\section{Driving experience}

Combining experiences of natural driving and race gaming affected gaze location strategy of drivers in video game context. Drivers-gamers showed larger relative fixations durations on the race lane (i.e., a relevant area of the visual scene) compared to non-driversgamers. This result partially confirms this study main hypothesis about the effects of natural driving practice on gaze behaviour of gamers. In car driving, visual cues from lane provide essential information to the steering control (Land and Horwood 1995; Land and Lee 1994). In addition, previous study evidenced that, in a simulated driving task, non-drivers-gamers ignored traffic road signs and relevant areas of interest compared to drivers-gamers (Ciceri and Ruscio 2014). It has been shown that playing racing video games enhance oculomotor performance and visual abilities of regular gamers compared to non-gamers (Castel, Pratt, and Drummond 2005; Dye, Green, and Bavelier 2009). However, when comparing experienced gamers with and without natural driving practice in a racing video game task, the 
gaze location strategy was adapted by the experience due to licensing process. Regarding to the performance, the results revealed non-significant difference between drivers-gamers and non-drivers-gamers during the racing task. One possible explanation for the similarity between drivers-gamers and non-drivers-gamers on performance outcome is related to the familiarization of both groups with the video game environment. In both groups, the experienced gamers reported a regular race gaming practice at the minimum frequency of three times per week with one hour per session. Therefore, the driving experience in natural context does not seem to account for improvement of performance of gamers in a racing video game task.

\section{Gaming experience}

Combining experiences of natural driving and race gaming affected gaze location strategy of drivers in video game context. Drivers-gamers more often inspections on the speedometer were accompanied by faster performance during the racing task compared to the drivers-non-gamers. This result confirms this study main hypothesis about the effects of race gaming experience on gaze strategy and performance of drivers. The superior performance among drivers-gamers was associated with increased monitoring of the speedometer. It seems that the attention of the drivers-gamers was shifted toward task-relevant information, arguably in an attempt to consciously adhere to speed instructions. This result corroborates previous findings according to which playing video games provides an improvement in visual attention and oculomotor performance of users (Castel, Pratt, and Drummond 2005; Dye, Green, and Bavelier 2009). Although both groups had similar experience in natural driving context, drivers-non-gamers were unable to seek for useful information in relevant areas of the visual scene in a racing video game task. It seems important to consider that the experimental task of this study was performed in a video game environment. In this case, the 
drivers-gamers were more familiar with the racing lane than the drivers-non-gamers and, therefore, they were more committed to the task instructions and drove as fast as possible. The level of realism of the racing game is also an aspect to be considered for the understanding of the drivers-non-gamers behaviour. In the current study, the racing video game was configured by means that the aspects of the game scenario could represent, or at least be as close as possible, the real-world driving environment. For instance, the players' viewpoint was positioned inside the vehicle rather than the third person, the presence of driving accessories such as steering wheel, brake and accelerator pedals, as well as the speedometer, and the use of high quality visual characteristics such as texture, luminosity and colours provided to the game content a highly realistic environment to the drivers. It is still controversial if the virtual environment can present a high level of fidelity compared to the natural world environment. Van Leeuwen and colleagues (2015) investigated the effects of visual fidelity on driving performance and gaze behaviour in a driving simulator study. The high fidelity level scenario (e.g., textured environment) resulted in higher mean driving speeds and increased horizontal gaze variance than the lower fidelity levels (van Leeuwen et al. 2015). On the other hand, it has been argued that lower visual fidelity driving simulator can be competent in driver training, in addition to having economic advantages compared to highly realistic simulation (Dahlstrom et al. 2009). In the current study, the essential characteristics of visual information provided by the virtual environment appeared to be quite similar to those available in a natural driving context (e.g., optic flow and perception of depth); since that all drivers-non-gamers were able to follow to the task instructions and they performed the task. It seems that the slower performance in the racing task by the driversnon-gamers is due to the lack of racing video game practice rather than the low visual fidelity of the virtual scenario. Further investigations are needed to examine the behaviour of racing 
gamers in driving simulators with complex road interactions and a highly realistic urban environment.

The combination of experiences in natural driving and race gaming provided the allocation of the gaze towards areas of interest in the visual scene in order to obtain taskrelevant information. The driving training in a virtual environment (e.g., race gaming and/or car driving simulators) might be aligned with the experience in natural driving context. Further investigations are needed to understand the relationship between driving experience in real-life and in racing video games and its effects of perceptual and motor behaviour. Particularly, the use of racing video games as an alternative training in the licensing learning and the natural driving practice for novice drivers, anxious drivers, elderly with or without a neurodegenerative disease (e.g., Parkinson's disease, Alzheimer) who still maintain the driving license.

\section{Limitations of the research}

Although a full factorial design and availability of baseline for all factors (e.g., a group of non-drivers-non-gamers) may be desirable, the comparison between drivers-nongamers and non-drivers-gamers groups was not intended. Here the approach was focusing on the combined driving-gaming experience (drivers-gamers group), testing for removal of each individual experience factor: gaming experience (drivers-non-gamers group) and driving experience (non-drivers-gamers group). In addition, there was a lack of subjective measure data on participants' level of immersion in the game and/or how realistic did the users judge the gaming scenario. This information could illustrate the debate on visual perception while driving and effects of gaming/driving experience. We plan for future studies focusing on pupillometry analyses as a way of discussing mental workload and a variety of potential effects of interest, such as attentional task requirement and immersion in the game. 


\section{Acknowledgements}

This work was supported by the São Paulo Research Foundation (FAPESP) Brazil to the Gisele Chiozi Gotardi under Grant [number 2015/10851-3]; and National Council for Scientific and Technological Development (CNPq) Brazil to the Sérgio Tosi Rodrigues under Grant [number 458916/2014-5].

\section{References}

Beullens, K., K. Roe, and J. Van Den Bulck. 2011. “Excellent Gamer, Excellent Driver? The Impact of Adolescents' Video Game Playing on Driving Behavior: A Two-Wave Panel Study." Accident Analysis and Prevention 43 (1). Elsevier Ltd: 58-65. doi:10.1016/j.aap.2010.07.011.

Castel, A. D., J. Pratt, and E. Drummond. 2005. "The Effects of Action Video Game Experience on the Time Course of Inhibition of Return and the Efficiency of Visual Search.” Acta Psychologica 119 (2): 217-30. doi:10.1016/j.actpsy.2005.02.004.

Ciceri, M. R., and D. Ruscio. 2014. "Does Driving Experience in Video Games Count? Hazard Anticipation and Visual Exploration of Male Gamers as Function of Driving Experience.” Transportation Research Part F: Traffic Psychology and Behaviour 22. Elsevier Ltd: 76-85. doi:10.1016/j.trf.2013.11.001.

Clarke, D. D., P. J. Ward, and W. A. Truman. 2005. "Voluntary Risk-Taking and Skill Deficits in Young Driver Accidents." Accident Analysis and Prevention 37 (3): $523-$ 529.

Cohen, J. 1988. Statistical Power Analysis for the Behavioral Sciences. 2nd editio. Hillsdale, NJ: Lawrence Erlbaum Associates, Inc.

Craig, Cathy. 2014. "Understanding Perception and Action in Sport : How Can Virtual Reality Technology Help?” Sports Technology. Taylor \& Francis. doi:10.1080/19346182.2013.855224.

Crundall, D., and G. Underwood. 1998. "Effects of Experience and Processing Demands on Visual Information Acquisition in Drivers.” Ergonomics 41 (4): 448-58. doi:10.1080/001401398186937. 
Dahlstrom, N., S. Dekker, R. van Winsen, and J. Nyce. 2009. "Fidelity and Validity of Simulator Training." Theoretical Issues in Ergonomics Science 10 (4): 305-14. doi:10.1080/14639220802368864.

Dye, M. W. G., C. S. Green, and D. Bavelier. 2009. "Increasing Speed of Processing with Action Video Games." Current Directions in ... 18 (6): 321-26. doi:10.1111/j.14678721.2009.01660.x.Increasing.

Gatersleben, B., and H. Haddad. 2010. "Who Is the Typical Bicyclist?” Transportation Research Part F: Psychology and Behaviour 13: 41-48.

Green, C. S., and D. Bavelier. 2003. “Action Video Game Modifies Visual Selective Attention." Nature 423 (6939): 534-37. doi:10.1038/nature01647.

Green, C.S., and D. Bavelier. 2007. “Action-Video-Game Experience Alters the Spatial Resolution of Vision: Research Article.” Psychological Science 18 (1): 88-94. doi:10.1111/j.1467-9280.2007.01853.x.

Howard, Matt C. 2017. "A Meta-Analysis and Systematic Literature Review of Virtual Reality Rehabilitation Programs." Computers in Human Behavior 70. Elsevier Ltd: 31727. doi:10.1016/j.chb.2017.01.013.

IBOPE. 2014. "Pesquisa Games Pop.” Accessed July 10. http://www.ibope.com.br/ptbr/relacionamento/imprensa/releases/Paginas/Pesquisa-inédita-do-IBOPE-Media-sobregames-revela-o-perfil-dos-jogadores-de-videogame-no-Brasil.aspx.

Instituto de Pesquisa Econômica Aplicada. 2015. “Acidentes de Trânsito Nas Rodovias Federais Brasileiras: Caracterização, Tendências E Custos Para a Sociedade.” Relatório de Pesquisa.

http://www.ipea.gov.br/portal/images/stories/PDFs/relatoriopesquisa/150922_relatorio_ acidentes_transito.pdf.

Konstantopoulos, P., P. Chapman, and D. Crundall. 2010. "Driver's Visual Attention as a Function of Driving Experience and Visibility. Using a Driving Simulator to Explore Drivers' Eye Movements in Day, Night and Rain Driving." Accident Analysis and Prevention 42 (3). Elsevier Ltd: 827-34. doi:10.1016/j.aap.2009.09.022.

Land, M. F., and J. Horwood. 1995. "Which Parts of the Road Guide Steering?” Nature 377: 339-340.

Land, M. F., and D. N. Lee. 1994. "Where We Look When We Steer.” Nature 369 (6483): 
742-44. doi:10.1038/369742a0.

Leeuwen, P. M. van, C. Gómez, A. R. Jimenez, R. Happee, and J. C. F. Winter. 2015. "Effects of Visual Fidelity on Curve Negotiation, Gaze Behaviour and Simulator Discomfort.” Ergonomics 58 (8): 1347-64. doi:10.1080/00140139.2015.1005172.

Mack, D. J., and U. J. Ilg. 2014. "The Effects of Video Game Play on the Characteristics of Saccadic Eye Movements." Vision Research 102. Elsevier Ltd: 26-32. doi:10.1016/j.visres.2014.07.010.

McDermott, A. F., D. Bavelier, and C. S. Green. 2014. "Memory Abilities in Action Video Game Players." Computers in Human Behavior 34. Elsevier Ltd: 69-78. doi:10.1016/j.chb.2014.01.018.

Neumann, David L, Robyn L Moffitt, Patrick R Thomas, Kylie Loveday, David P Watling, Chantal L Lombard, Simona Antonova, and Michael A Tremeer. 2017. "A Systematic Review of the Application of Interactive Virtual Reality to Sport.” Virtual Reality. Springer London. doi:10.1007/s10055-017-0320-5.

Pasco, Denis. 2013. “The Potential of Using Virtual Reality Technology in Physical Activity Settings.” Quest 65: 429-41. doi:10.1080/00336297.2013.795906.

Underwood, G., P. Chapman, N. Brocklehurst, J. Underwood, and D. Crundall. 2003. "Visual Attention While Driving: Sequences of Eye Fixations Made by Experienced and Novice Drivers.” Ergonomics 46 (6): 629-46. doi:10.1080/0014013031000090116.

Underwood, G., D. Crundall, and P. Chapman. 2007. "Driving.” In Handbook of Applied Cognition, edited by T. D. Francis, 2th ed. John Wiley \& Sons, Ltd.

West, G. L., N. Al-Aidroos, and J. Pratt. 2013. “Action Video Game Experience Affects Oculomotor Performance.” Acta Psychologica 142 (1). Elsevier B.V.: 38-42. doi:10.1016/j.actpsy.2011.08.005.

World Health Organization. 2016. "Road Traffic Injuries.” Media Center. http://www.who.int/mediacentre/factsheets/fs358/en/. 


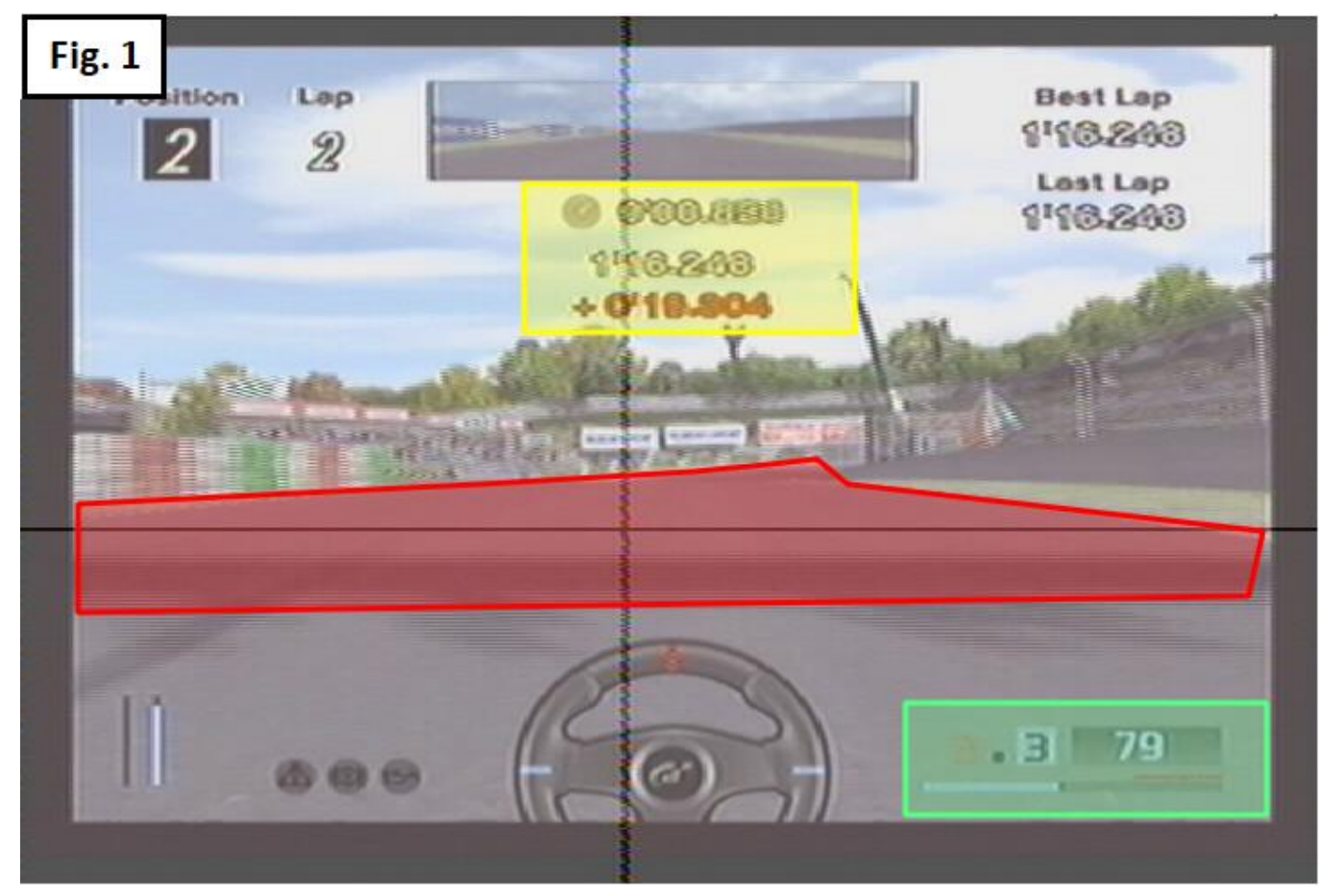

Figure 1. The scene video from Eye Tracker that represents the participant's line of gaze (black cursor). AOIs defined for data analysis: race lane (red), chronometer (yellow), speedometer (green), and outside (all part of the visual scene that was not considered one AOI).

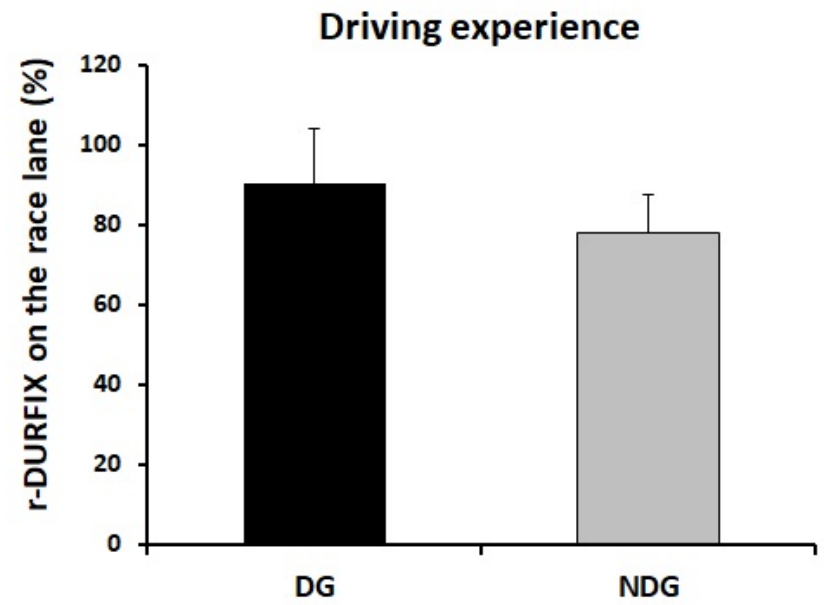

Figure 2. Relative fixations duration $(\%)$ on the AOI race lane for the driving experience comparison (drivers-gamers vs. non-drivers-gamers). 


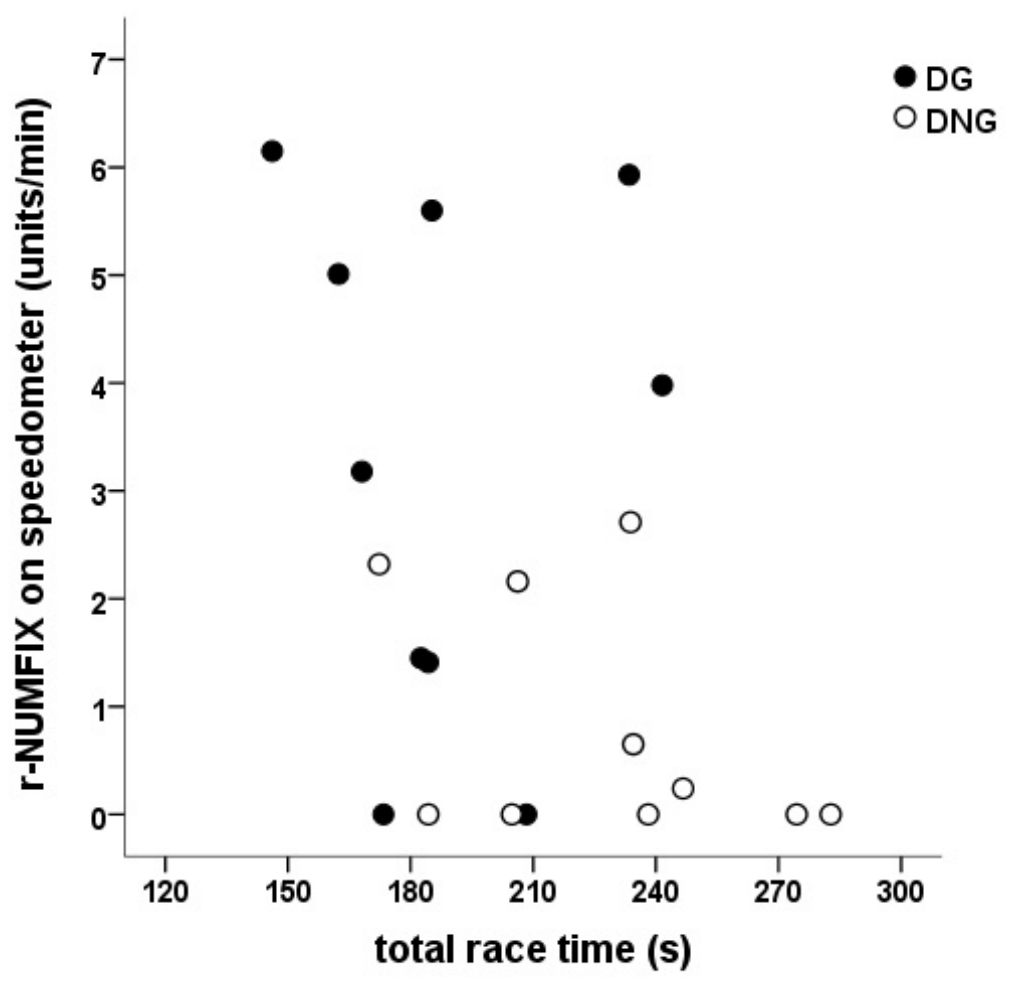

Figure 3. Relative number of fixations (axis y) by total race time (axis $\mathrm{x}$ ) for the gaming experience comparison (drivers-gamers vs. drivers-non-gamers). 
Table 1. Means (SDs) of the relative number and duration of fixations per AOI, variance of fixations location in horizontal and vertical axes, and the performance for both driving and gaming experience comparisons $(\mathrm{n}=30)$.

\section{Gaming experience Driving experience}

DNG DG NDG

\section{Outside}

r-NUMFIX (units/min)

$1.75(2.43)$

$1.43(1.17)$

$2.17(1.29)$

r-DURFIX (\% trial time)

$0.91(1.78)$

$0.59(0.50)$

$0.78(0.46)$

Race lane

r-NUMFIX (units/min)

$138.09(14.80)$

$137.48(28.30)$

131.02 (13.02)

r-DURFIX (\% trial time)

86.19 (6.59)

$90.30(13.61)$

$78.13(9.60)^{*}$

Chronometer

r-NUMFIX(units/min)

$1.91(3.23)$

$3.07(3.34)$

$5.31(3.99)$

r-DURFIX (\% trial time)

$1.20(2.43)$

$1.57(2.06)$

$3.10(2.50)$

Speedometer

r-NUMFIX (units/min)

$0.80(1.12)^{\dagger}$

4.27 (4.17)

$3.49(4.33)$

r-DURFIX (\% trial time)

$0.37(0.54)$

$1.95(2.36)$

$1.64(2.25)$

Variance of Fixations

Horizontal axis (pixels)

$1099.56(357.82) \quad 1079.92(476.50)$

$1040.40(401.59)$

Vertical axis (pixels)

$293.14(108.13)$

$424.59(237.19)$

$585.01(311.97)$

Performance

Total race time (s)

$198.90(36.31)$

r-NUMFIX: relative number of fixations; r-DURFIX: relative fixations duration; DG:

drivers-gamers; DNG: drivers-non-gamers; NDG: non-drivers-gamers; * significant group main effect for driving experience $(*$ vs. DG); $\uparrow$ significant group main effect for gaming experience († vs. DG). 